\title{
Technical Note: How image processing facilitates the rising bubble technique for discharge measurement
}

\author{
K. P. Hilgersom and W. M. J. Luxemburg \\ Water Resources Section, Faculty of Civil Engineering and Geosciences, Delft University of Technology, P.O. Box 5048, \\ 2600 GA Delft, The Netherlands
}

Correspondence to: K. P. Hilgersom (k.p.hilgersom@tudelft.nl)

Received: 9 September 2011 - Published in Hydrol. Earth Syst. Sci. Discuss.: 20 September 2011

Revised: 21 January 2012 - Accepted: 1 February 2012 - Published: 6 February 2012

\begin{abstract}
In this article, we rehabilitate the integrating rising bubble technique as an effective means of obtaining discharge measurements. Since Sargent (1981, 1982a), the technique has not been applied widely, mainly as a result of practical difficulties. We hypothesize that modern image processing techniques can greatly improve the rising bubble technique. We applied the technique in both a laboratory setup and a field study, after determining the bubble rising velocity for our nozzles in the specific case. During our measurements, we captured digital photographs of the bubble envelope at the water surface, each picture being a single measurement of the discharge. The photographs were corrected for lens distortion and reprojected so that accurate distances on water surface level could be obtained. This easy digital procedure resulted in accurate discharge measurements, even when turbulence was involved and the averages of multiple image analyses yielded good results. The study shows that the rising bubble technique can be a preferable discharge gauging technique in some situations. Recent developments in image processing facilitate the method substantially.
\end{abstract}

\section{Introduction}

A large variety of discharge gauging techniques is applied in the field of hydrological measurements. Each of them performs best under specific stream properties, such as the geometry of the cross-section and turbulence. The integrating float method, one of these techniques, best resembles the frequently applied method using a float at the water surface. With the latter method, hydrologists measure stream flow by releasing a float at the water surface and calculating the velocity from its displacement over time. This method is easy and quick but is considered relatively inaccurate, for an uncertain correction factor is applied to estimate the depthaveraged velocity from the measured surface velocity.

To overcome this, the integrating float method has been developed (Herschy, 1978, 1995), where a float is released at the bottom of a river or canal. The float is assumed to rise with a constant velocity, so the depth-integrated horizontal velocity can be determined from the float's displacement as it surfaces. Air bubbles are a type of float that can be applied in a simple manner. This article discusses the merit of modern computational and photogrammetric techniques for applying the integrating float method and specifically the rising bubble technique to measure river discharge (Sargent, 1981; Shaw, 1994).

Already in the 19th century, D'Auria described a method in which a sinker was released at water surface level to determine the depth-integrated velocity by the total horizontal displacement of the sinker as it reaches the river bed (Sargent, 1981). At the start of the twentieth century, Hajos (1904) described the reverse procedure: floats are released at the stream bed and their displacement can be found when reaching the surface. Amongst others, Liu and Morris (1970) improved this method and investigated the influence of turbulence on the method.

Viol and Semenov (1964) were the first to apply this integrating float method using air bubbles as floats, assuming these air bubbles have a constant rising velocity. Their work formed the basis for research by Sargent (1981, 1982a,b), who applied two photo cameras with autowinders to obtain a time series of photographs of surfacing air bubbles. The horizontal displacements of the air bubbles with respect to their release points on the river bed could then be calculated from the two pictures taken from different angles using a standard photogrammetric technique. Repeating the procedure provided an easy means to register how stream discharge evolves over time (Sargent, 1981, 1982a). Sargent's work meant a large advance with respect to applicability and 
accuracy of the method. However, since Sargent's publications, the method has only been applied by a few authors (Toop et al., 1997; Yannopoulos et al., 2008), suggesting that previous technologies made the method less easy and more expensive to apply compared to other gauging methods. A discussion of Sargent's work confirms this (Sargent and Davis, 1981). We believe that modern techniques can significantly improve the rising bubble technique.

To address the potential of the rising bubble technique as a modern and usable method, we introduce the application of image processing techniques. With this novel approach, we obtain quantitative data from photographs that were taken during tests in a laboratory flume, and case studies in the channelized river Reitdiep (The Netherlands) and the river Sûre (Grand Duchy of Luxembourg). We demonstrate that the use of a single photo or video camera is sufficient to perform accurate measurements and that automation of the process provides the opportunity to perform continuous measurements with this gauging technique. Measurement results from the case study show that the method performs accurately in the specific situations.

Rather than a manual on measuring with the rising bubble technique, the aim of this publication is to show how the addition of digital image processing techniques supports the method.

\section{Study site}

We tested the rising bubble technique both in a laboratory flume and in the field. The field study consisted of measurements in two distinct situations: a lock with a rectangular cross-section and and a natural stream with a more variable cross-section.

\subsection{Lock measurements}

The first field study was performed in the Provinciale Sluis $\left(53^{\circ} 20^{\prime} 14^{\prime \prime} \mathrm{N}, 6^{\circ} 17^{\prime} 50^{\prime \prime} \mathrm{E}\right)$, a lock near the village of Zoutkamp (province of Groningen, The Netherlands), on 9 October 2009. This 9-meter-wide lock is constructed on the point where the channelized river Reitdiep enters the Lauwersmeer (see Fig. 1). At the time that this lake was still part of the Wadden Sea, the lock allowed ships to transfer between the tidally influenced sea level and the controlled water level of the Reitdiep. Since the Lauwersmeer was dammed in 1969, the lock is no longer required. Nevertheless, its rectangular cross-section makes the lock suitable to accurately perform automatic discharge measurements. For this reason, the lock is equipped with an acoustic discharge measurement device (ADM). Both the availability of data from the ADM and the lock's geometry make this site suitable for first testing the rising bubble technique. Besides, the bridge above the lock makes it possible for us to take photographs from above the water surface.

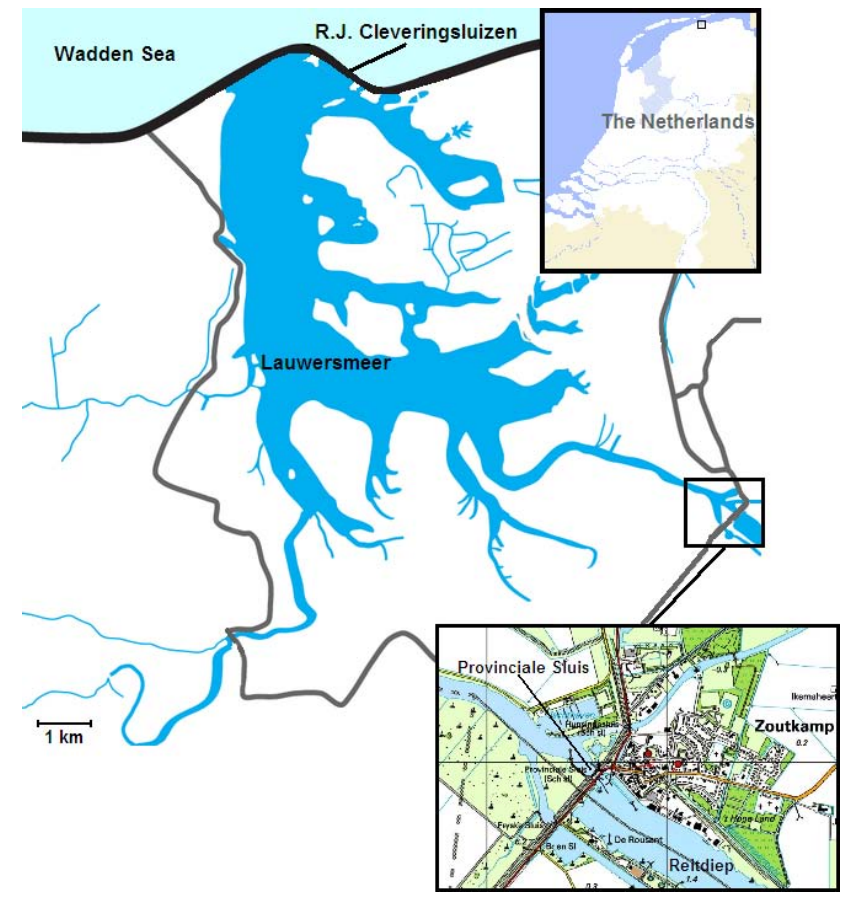

Fig. 1. Map of the Lauwersmeer; the dark grey lines represent the surrounding embankment.

The Lauwersmeer discharges into the Wadden Sea during low tide at sea by means of the R. J. Cleveringsluizen. Opening these sluices strongly enhances the discharge of the Reitdiep near Zoutkamp. To apply the rising bubble technique at varying stream discharges, the R. J. Cleveringsluizen were opened during part of our field measurements.

\subsection{Natural stream measurements}

We studied the technique's performance for a natural stream in the Sûre River (Grand Duchy of Luxembourg) on 10 May 2011. The study site is located between the villages of Boulaide and Bigonville, upstream from the lake Lac de la Haute-Sûre (location: $49^{\circ} 51^{\prime} 58^{\prime \prime} \mathrm{N}, 5^{\circ} 48^{\prime} 22^{\prime \prime} \mathrm{E}$ ). Locally, the river is $12.5 \mathrm{~m}$ wide and has a natural bed that is shallow on the right half and deeper on the left (facing downstream).

\section{Materials and methods}

\subsection{Method}

The principle of the integrating float method is that the time $T$ needed for a float to surface with $v_{z}$ equals the time needed for the float to displace over distance $L$ with a depthaveraged horizontal velocity $\overline{v_{x}}$ (see Fig. 2):

$T=\frac{D}{v_{z}}=\frac{L}{\overline{v_{x}}}$, 


\section{Longitudinal cross-section}

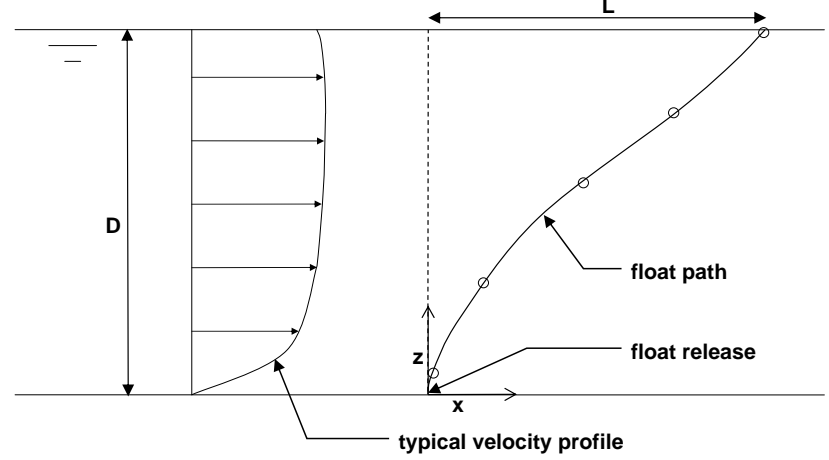

Fig. 2. Float path occurring with a typical velocity profile over depth.

where $D$ denotes the vertical distance between streambed and water surface. From Eq. (1), we find for the specific discharge $q$ (i.e. the depth-integrated horizontal velocity):

$q=\int_{0}^{D} v_{x} \mathrm{~d} z=\overline{v_{x}} \cdot D=v_{z} \cdot L$,

as is described by Sargent (1982a). Equation (2) shows that the specific discharge can be calculated from the constant $v_{z}$ and the displacement of the float while surfacing $(L)$. The latter parameter varies over the width of the stream $(y$ direction), and several floats are needed to determine $L(y)$. Integrating the measured displacements leads to the total discharge $Q$ :

$Q=\int_{0}^{w} q \mathrm{~d} y=v_{z} \cdot \int_{0}^{w} L(y) \mathrm{d} y=v_{z} \cdot A$,

$A$ being the surface of the bubble envelope (see Fig. 3 ).

\subsection{Application}

For the rising bubble technique, air bubbles are the specific floats. Air bubbles are easier to release from the bottom of the stream compared to other, rigid floats. Furthermore, they make the performance of a series of measurements less complicated.

Air bubbles usually appear at the surface as air bubble clusters. In those cases, the surfacing locations of the bubbles are best indicated by the center of the visible rings on these spots. After surfacing, air bubbles remain visible while continuing with the flow. This leaves traces of air bubbles on the water surface, like are shown in Fig. 3. Of interest for this method are the air bubbles, or the centers of air bubble clusters, that are the closest to the release points.

We first tested the method in a laboratory flume. We used two nozzles to release the air bubbles, one nozzle being located near the wall of the flume and one in the middle (see Fig. 4). They were connected to compressed air by small tubes and a pressure regulator controlled the air pressure to
Top view

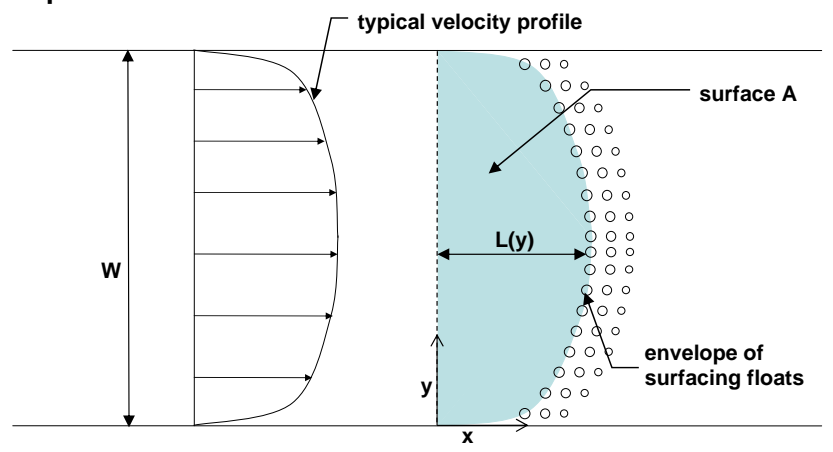

Fig. 3. Surface $A$ between the float release line and the envelope of surfacing floats.

just above the water pressure at the bottom of the flume. The nozzles were short steel pipes (diameter: $22 \mathrm{~mm}$ ) with a $1 \mathrm{~mm}$-wide hole punched into the wall and a click connector on one side of the pipe for the entering air tube. The steel pipes were thickened around the hole to a thickness of $5.6 \mathrm{~mm}$ to produce more consistently sized air bubbles (this effect of thicker walls was found in a laboratory test).

We assumed that the horizontal flow profile is symmetrical. We registered the displacements of the air bubbles both by a digital photo camera (Nikon D60; lens: Nikkor AF-S 18-55 $\mathrm{mm} \mathrm{f/3.5-5.6} \mathrm{G} \mathrm{ED} \mathrm{DX} \mathrm{-} \mathrm{this} \mathrm{combination} \mathrm{was} \mathrm{used}$ in all the case studies) and measuring tape, the latter being a reference for the accuracy of the displacements according to the processed photographs. Later, we will describe how the photographs were processed. We compared the discharges found from the photos with data from an Endress + Hauser Prosonic Flow 91W acoustic flow meter that was mounted to the entering pipe of the flume.

In our field study in the lock near Zoutkamp, we chose to lay a steel pipe (diameter: $22 \mathrm{~mm}$ ) across the stream. The reason for this is that the lock had a flat floor and the flow might unequally displace separate nozzles (which would not be noticed from the top of the water surface). The pipe had holes with a diameter of $1 \mathrm{~mm}$ punched in every $75 \mathrm{~cm}$ to release small air bubbles. Practicalities prevented us from thickening the pipe around the nozzle hole, as was done in the other situations.

One end of the pipe was connected to an air compressor via a corner piece and a standing pipe. We chose this construction, since the standing pipe held the horizontal pipe in upright position (i.e. the holes remained facing upward). We adjusted the air pressure in the pipe to just above the water pressure at the bottom of the stream. Figure 5 shows a schematic overview of the measurement setup. On locations where no electrical power is available, a cylinder of compressed air can replace the air compressor.

We registered the displacement of the air bubbles with a digital camera from a bridge that is more than $4 \mathrm{~m}$ above the 


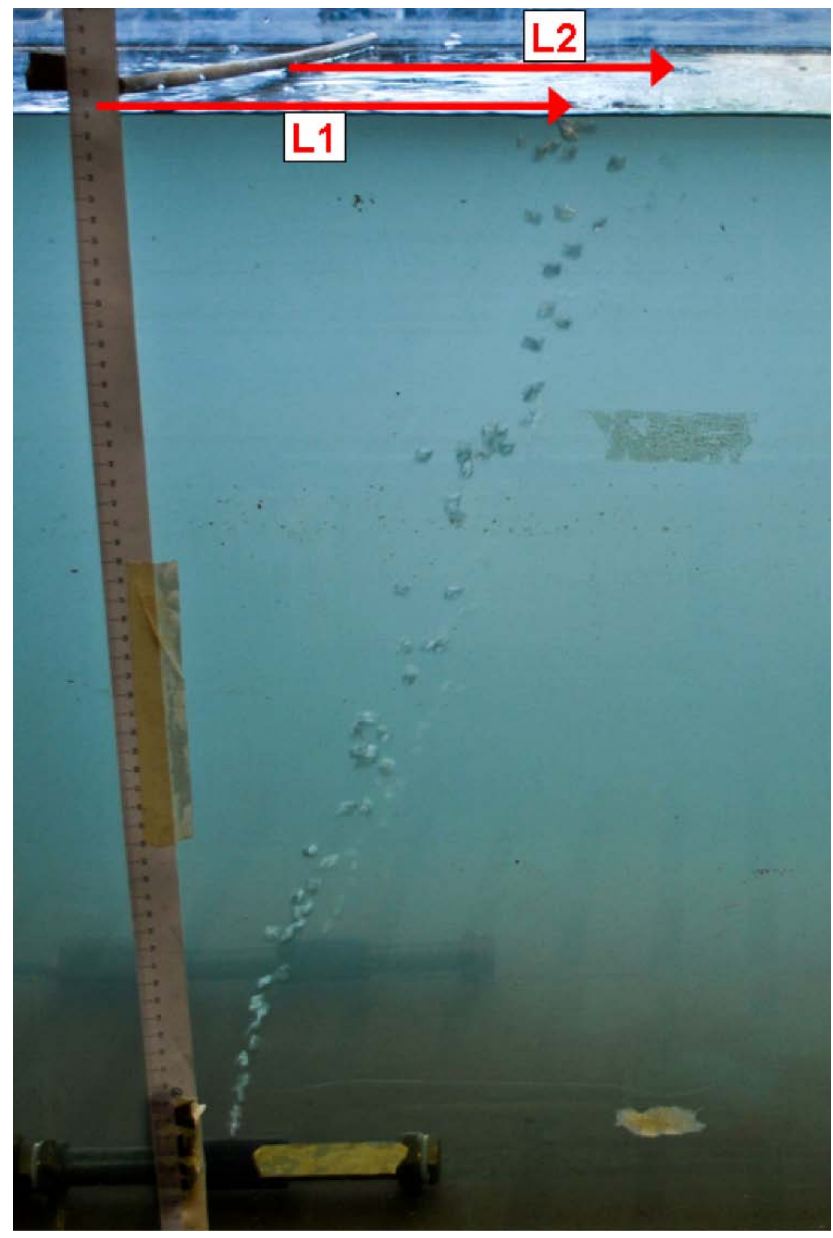

Fig. 4. Side view of the measurements in the laboratory flume; $L 1$ represents the horizontal displacement of bubbles originating from the nozzle located near the flume's wall, $L 2$ of the ones from the nozzle located in the middle.

water surface. We made sure we captured the location of the pipe, the bubble envelope, and four reference points that were needed for the image processing all in one image. From the processed images, we could find the discharges over time and compare them with reference measurements from the acoustic discharge measurement device located in the lock (Elster Instromet, FLOW-2000 model 5; the device uses the travel times of acoustic signals between two pairs of transducers to calculate the longitudinal and transversal velocity in the stream at one representative depth). In addition, we took a reference measurement with a propeller current meter (Valeport, Model BFM002) at three depths for three verticals in the cross-section.

In the Sûre River, the use of a steel pipe was made impossible by the varying depth of the river bed. We chose to place five nozzles on the bed of the same type as applied in the laboratory flume. They were connected to a cylinder of compressed air by small tubes (no electricity was available at this

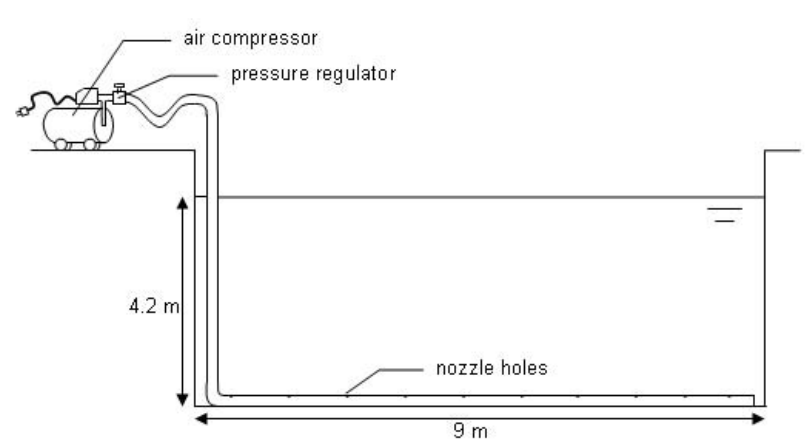

Fig. 5. Situation sketch for the measurements in the lock near Zoutkamp.

site), two of the nozzles being located on the shallow side of the cross-section (ca. $30 \mathrm{~cm}$ deep) and the other three in the deeper part (ca. $60 \mathrm{~cm}$ deep). Three pressure regulators controlled the air pressure to just above the water pressure at bed level. An attached tile prevented the nozzles from floating and kept the hole facing upwards.

We used a step ladder on one bank of the river to take photos from a sufficient level above the water surface. Four traffic cones with measured relative distances, two on each bank, marked our reference points. Ideally, the bases of these traffic cones are on water surface level. In this case, we placed two of the traffic cones a bit higher due to the local shape of the banks. Estimating the locations of these cones as if they were on water surface level might have introduced an additional small error for this case study. Again, the pictures were taken so that all the reference points and the complete bubble envelope were captured. A measurement with a propeller current meter (SEBA-Mini Current Meter M1, propeller serial no. 250257; the measurements were performed in six verticals and at three depths) constituted our reference measurement.

\subsection{Image processing}

The novelty of this research consists for a large part of applying digital image processing to the integrating float method. The following will describe the subsequent steps to obtain area $A$ from a digital photograph. We performed the processing using the Image Processing Toolbox of Matlab ${ }^{\circledR}$, because the toolbox provides part of the tools we need and the Matlab scripting makes the process easy to repeat.

The photograph must fully capture the bubble profile and the reference points, and surfacing bubbles must be visible (both in terms of size on the picture and light circumstances). The reference points are used to transform the image to a 2-D projection. They should be on the water surface level and have known relative distances.

A photo camera lens usually deforms photographs due to a varying shape and an imperfect centering, meaning that 
photos cannot be used straightaway. This is called optical aberration and affects the photograph by radial and tangential distortion (Brown, 1966). Tangential distortion, caused by imperfect centering of lenses, is negligible in contemporary cameras (Tsai, 1987; Nowakowski and Skarbek, 2007). Therefore, we will correct our images for radial distortion (i.e. the displacement of pixels into radial direction from the center of distortion).

Radial distortion appears mainly in the form of barrel or pincushion distortion (De Villiers et al., 2008; Neale et al., 2011). In the former, the image looks bulged, and in the latter it looks squeezed. In most cases however, camera lenses appropriate for this method will cause barrel distortion.

The radial symmetric nature of barrel distortion makes it easy to correct a picture for this distortion, as is often done in the practices of architectural photography and 3-D computer vision, where camera lenses bend for example the straight lines of buildings (Devernay and Faugeras, 1995, 2001; Stein, 1997; Swaminathan and Nayar, 2000), and in photogrammetry. The latter is the practice of determining the geometric properties of objects in pictures and is the major concern of this research. A simple algorithm for correcting for barrel distortion (i.e. for squeezing the "bulged" photo back) is based on the following equation (Brown, 1971; Fryer and Brown, 1986):

$r_{u}=r_{d}+k_{1} \cdot r_{m d}^{3}+k_{2} \cdot r_{d}^{5}+k_{3} \cdot r_{d}^{7}+\ldots$,

where $r_{u}$ and $r_{d}$ are the radii for each pixel relative to the center of distortion (normalized to the maximum radius at the corners) for the undistorted and the distorted picture, respectively. Since we may assume that barrel or pincushion distortion constitutes most of the lens' radial distortion, the third order term is predominant in Eq. (4) (Brown, 1971), and allows us to rewrite the equation:

$r_{u}=r_{d}+k_{1} \cdot r_{d}^{3}$.

The constant $k_{1}$ is lens-specific and is often provided by manufacturers or specialized websites, but one can also calibrate it oneself.

The procedure maps input pixels from their original locations (denoted by integer coordinates) to new coordinates that are unlikely to have integer coordinates. The subsequent resampling of output pixels leads to minor information losses (De Villiers et al., 2008). Step (a) in Fig. 6 represents the correction for lens distortion.

Afterwards, we morph the images to a horizontal (2-D) plane at the water surface level, using the four selected reference points. Theory about this technique follows from literature about Digital Image Warping, a subfield of Digital Image Processing (Wolberg et al., 1990). There are several software packages that support the projective warping of images. We use Matlab's Image Processing Toolbox to set up a transformation array on which we map our photograph.

A projective transformation of a quadrilateral to a quadrilateral requires four reference points which should in our case be located on the level of the water surface, since that is the plane from which we want to obtain distances. The four reference points are not required to be the corners of a rectangle. The result of the transformation shows large distortion of objects outside this plane as shown by step (b) (Fig. 6). Within the plane, on the other hand, a 2-D image of the water surface correctly represents the distances.

Like in step (a), the resampling of output pixels in step (b) causes minor information losses, resulting in a possible deterioration of the visibility of surfacing air bubbles as the photograph passes through steps (a) and (b). Since step (c) determines the bubble envelope based on the locations of the bubbles, we choose to mark the points of interest (i.e. the locations of the air bubbles and the nozzle line) in the unprocessed photograph, and recalculate their positions as the photograph passes through the first two steps.

Step (c) in Fig. 6 interpolates the area between the nozzle line and the bubble envelope. Since Matlab used the known relative distances of the reference points when recalculating the locations of the release pipe and the air bubbles at the water surface, these locations are properly georeferenced. From this it is an easy step to calculate the area enclosed between the nozzle line on the river bed and the bubble envelope at the water surface and, subsequently, the discharge according to Eq. (3).

When the stream cross-section is rectangular and the flow direction is approximately perpendicular to the cross-section, as is the case in the lock near Zoutkamp and in the flume, it is sound to assume a logarithmic shape of the transversal profile of flow velocities. Therefore, we interpolate the surfacing bubbles in the lock and the flume with a logarithm, whereas for the natural river a linear interpolation is applied.

\subsection{Rising velocity}

The rising bubble technique assumes a constant rising velocity $v_{z}$ of the air bubble that is released at the bottom of the stream. However, this assumption does not represent reality in every case. Several authors (e.g. Sargent, 1982b; Toop et al., 1997; Yannopoulos et al., 2008) have shown that applying this assumption depends on the air pressure, the size of the nozzle from which the air bubble is released, and water properties like turbulence, temperature and contamination. More detailed research on the motion of gas bubbles in a liquid has been performed by, amongst others, Aybers and Tapucu (1969a,b) and Lehrer (1980). These publications teach us that, for a certain range of air bubble diameters, the rising velocity does not change much with varying diameters. This means that proper air bubbles for the rising bubble technique should have sizes within this range of approximately 2 to $4 \mathrm{~mm}$, depending on the circumstances. Bubbles with these sizes have commonly an ellipsoidal shape (e.g. Wesselingh, 1987).

Before our discharge measurements, we conducted laboratory tests to investigate the rising velocity for several nozzle 


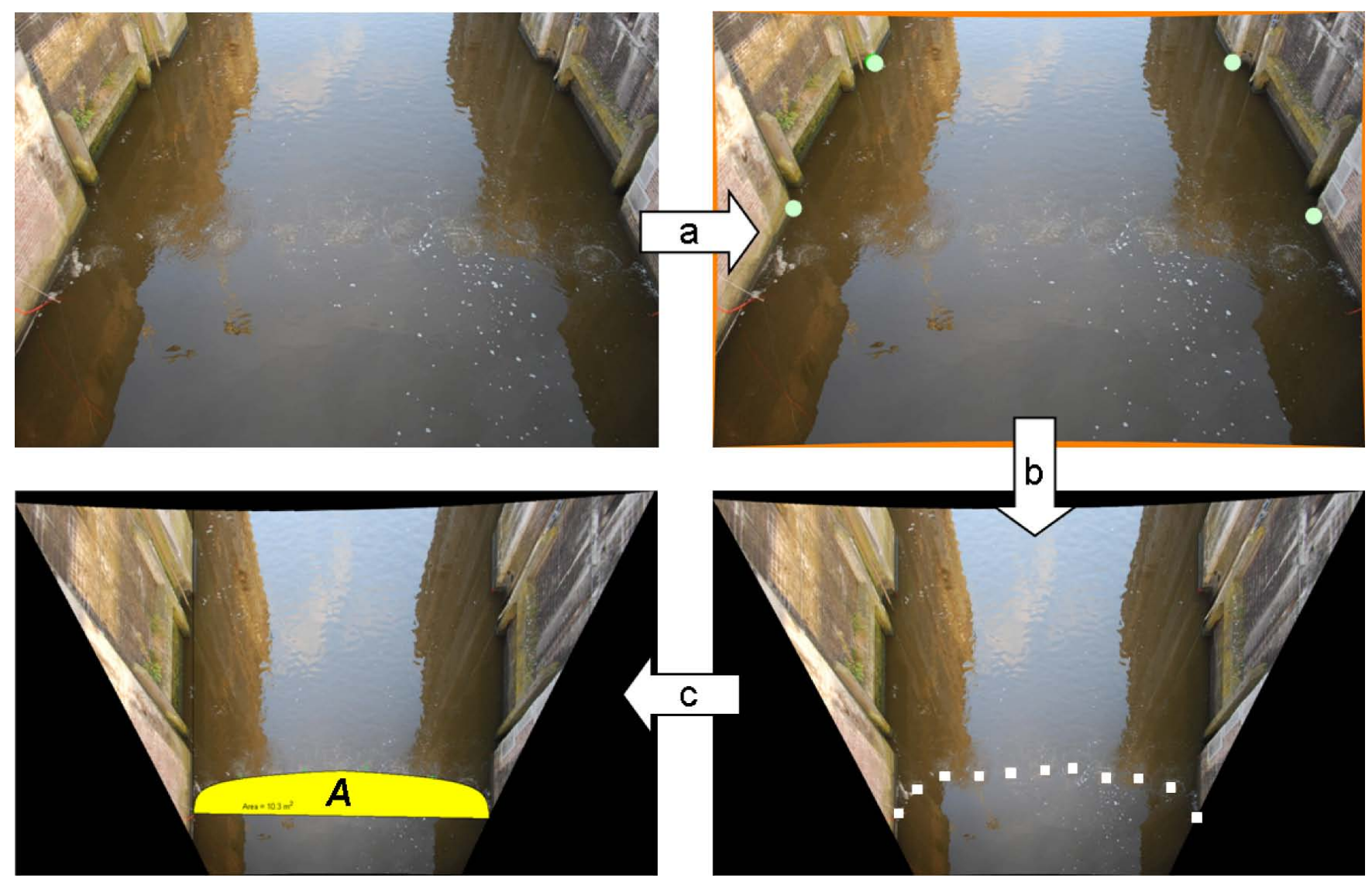

Fig. 6. Steps performed to process the image and obtain surface area $A$; step (a) represents the correction for lens distortion, step (b) represents the 2-D reprojection of the image based on the four reference points on a horizontal plane (the light green circles), and step (c) finds the area in between the nozzle line and the bubble envelope (the white squares).

sizes and air pressures. The tests were conducted inside a transparent, 2-meter-high column (diameter: $30 \mathrm{~cm}$ ) and compared the bubbles resulting from varying nozzle diameters, water levels and air pressures. From the results, we determined the nozzle design for our study.

Since water properties appeared to influence the rising velocity, we used a similar but smaller column in the field to measure this velocity for the specific situation. The column was filled to a level of $1.5 \mathrm{~m}$ to have enough distance to do accurate velocity measurements, based on the time that tracked air bubbles needed to pass through a distance of $1 \mathrm{~m}$. Since the size of the nozzles was determined so that depth effects to the rising velocity appeared minimal, we assumed the measured velocity to hold for other water depths as well. In the case of the flume measurements, we measured the rising velocity of the air bubbles both in the flume and the $1.5-\mathrm{m}$ column.

\section{Results and discussion}

\subsection{Rising velocity}

From our laboratory measurements of the rising velocity, we concluded that a nozzle opening of $1 \mathrm{~mm}$ in diameter together with an air pressure just above the water pressure around the nozzle produced air bubbles that are most likely within the range of 2 to $4 \mathrm{~mm}$. Within this range, air bubbles are commonly ellipsoidal and remain almost constant in vertical velocity while surfacing (e.g. Wesselingh, 1987). However, since the main focus of our research is the applicability of image processing techniques, further research is required to design a nozzle that produces air bubbles with consistent rising velocities.

We also found that a field determination of the rising velocity is currently still necessary to get a good estimate. For our discharge measurements in the laboratory flume, we observed both in the flume itself and in the column that $v_{z}$ was $0.23 \mathrm{~m} \mathrm{~s}^{-1}$, whereas for our field studies in the lock and in the river Sûre $v_{z}$ was $0.35 \mathrm{~m} \mathrm{~s}^{-1}$ and $0.32 \mathrm{~m} \mathrm{~s}^{-1}$, respectively. These differences can be attributed to different air pressures, 
water temperatures and contamination, as well as a minor change in nozzle design (a thickening of the wall of the steel pipe). We recommend further research on these influencing factors to better predict the rising velocity, given the governing circumstances. In the following, we use the rising velocities that we determined on site.

\subsection{Flume measurements}

We first applied the method in a laboratory flume. Table 1 shows that the rising bubble technique gave accurate results in the laboratory situation, measuring on average $47.11 \mathrm{~s}^{-1}$ by photo integration as compared to $46.61 \mathrm{~s}^{-1}$ measured by an acoustic flow meter in the entering pipe of the flume. Variations in the discharges measured, as illustrated by the variations in the distances $L 1$ and $L 2$ (see Fig. 4 ) as well as the average percentage deviation of five rising bubble technique measurements relative to their average value, can be attributed to the turbulent nature of the flow inside the flume. However, as the number of samples was increased by analyzing more photographs, the rising bubble technique provided consistent results.

\subsection{Lock measurements}

Data from the field study was obtained during a field study on 9 October 2009. During low tide in the Wadden Sea, the R. J. Cleveringsluizen were opened to vary the discharge in the Provinciale Sluis. This strongly influenced the bubble envelope at the water surface (see Fig. 7). Figure $8 \mathrm{com}$ pares the results we obtained from the rising bubble technique with the time series from the acoustic discharge measurement device. We performed one propeller current meter measurement as a reference. Since this method requires measurement of velocities at several locations across the crosssection, this is the most labor intensive of the three methods and was, therefore, performed only once. The transient flow makes the moment of performing the propeller current measurements rather unfortunate.

From our observations, the measurement error of the rising bubble technique compared to the acoustic discharge measurements appears on average about $13 \%$ at low discharges, whereas at higher discharges the measurement error reduces to $3 \%$ (see Table 2). We argue that the relative error at lower discharges is larger, since the relative error in determining the distance that a bubble travels $\left(r_{L}\right)$ is large when this distance $L$ is low compared to the measurement error $\triangle L\left(r_{L}=\triangle L / L\right)$.

\subsection{Natural stream measurements}

Table 3 shows the results for measurements in the natural river Sûre on 10 May 2011. The photos were taken on average every $10 \mathrm{~s}$. The low percentage errors show that the analysis of multiple photographs can lead to consistent results,
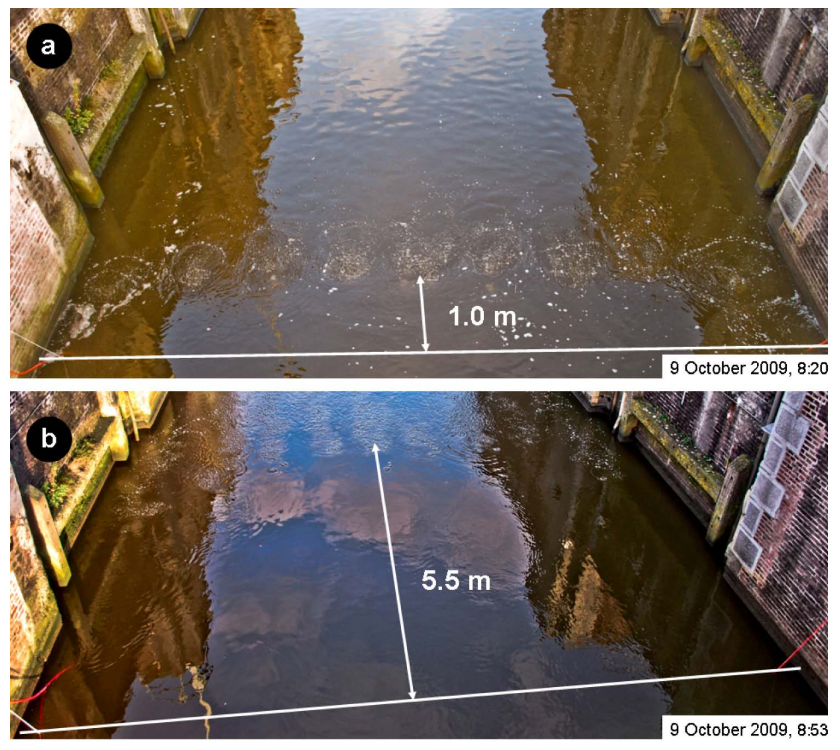

Fig. 7. The bubble envelope during low $\left(3.3 \mathrm{~m}^{3} \mathrm{~s}^{-1}\right)$ and high $\left(16 \mathrm{~m}^{3} \mathrm{~s}^{-1}\right)$ discharge, respectively.

Table 1. Discharges measured with the photogrammetric technique in a laboratory flume with a constant discharge; the absolute deviations are relative to the average discharge measured with the rising bubble technique; as a reference, hand measurements of the distances $L 1$ and $L 2$ (see Fig. 4) measured on average $25 \mathrm{~cm}$ and $27 \mathrm{~cm}$, respectively.

\begin{tabular}{lcccc}
\hline Measurement & $L 1(\mathrm{~cm})$ & $L 2(\mathrm{~cm})$ & $Q\left(1 \mathrm{~s}^{-1}\right)$ & Deviation \\
\hline 1 & 24.6 & 26.9 & 48.1 & $2.08 \%$ \\
2 & 23.8 & 29.7 & 47.4 & $0.59 \%$ \\
3 & 23.8 & 28.0 & 45.6 & $3.23 \%$ \\
4 & 27.2 & 25.1 & 48.5 & $2.93 \%$ \\
5 & 24.3 & 26.3 & 46.0 & $2.38 \%$ \\
\hline Average & 24.7 & 27.2 & 47.1 & $2.24 \%$ \\
\hline
\end{tabular}

even in this environment and with this limited amount of five nozzles.

In Photo 5, two of the surfacing bubble clusters were poorly visible, which may account for the relatively large deviation from the average of over $4 \%$. The poor visibility of surfacing air bubbles is both due to shading effects on the water surface (e.g. compare the visibility of the left and right surfacing location marked in Fig. 9a) and the low resolution of a zoomed surfacing bubble. The latter cause indicates that, for our type of photo camera, this river size is the maximum that the method can handle. However, a change in the setup, e.g. by hanging the camera in the middle above the stream, can double the maximum river width for which this method works. A higher-resolution camera can also expand this range. 
Table 2. Absolute percentage deviations for the discharges $(Q)$ measured with the rising bubble technique (RBT) compared with the acoustic discharge measuring device (ADM) in Zoutkamp (9 October 2009); cross-sectionally averaged velocities $\bar{v}$ lower then $0.2 \mathrm{~m} \mathrm{~s}{ }^{-1}$ are considered low.

\begin{tabular}{lcccc}
\hline Time CET & $\overline{v_{\mathrm{RBT}}\left(\mathrm{m} \mathrm{s}^{-1}\right)}$ & $Q_{\mathrm{RBT}}\left(\mathrm{m}^{3} \mathrm{~s}^{-1}\right)$ & $Q_{\mathrm{ADM}}\left(\mathrm{m}^{3} \mathrm{~s}^{-1}\right)$ & Deviation \\
\hline $07: 14$ & 0.07 & 2.4 & 2.9 & $14.9 \%$ \\
$07: 34$ & 0.07 & 2.8 & 3.1 & $9.7 \%$ \\
$07: 47$ & 0.06 & 2.3 & 2.8 & $19.8 \%$ \\
$08: 15$ & 0.06 & 2.2 & 2.5 & $11.0 \%$ \\
$08: 18$ & 0.08 & 2.9 & 2.6 & $10.8 \%$ \\
$08: 21$ & 0.10 & 3.6 & 3.0 & $17.3 \%$ \\
$08: 22$ & 0.10 & 3.8 & 3.4 & $11.3 \%$ \\
$08: 26$ & 0.14 & 5.3 & 4.8 & $11.6 \%$ \\
$08: 40$ & 0.30 & 11.1 & 10.6 & $4.7 \%$ \\
$08: 43$ & 0.33 & 12.3 & 11.9 & $3.5 \%$ \\
$08: 45$ & 0.34 & 12.6 & 12.8 & $1.7 \%$ \\
$08: 50$ & 0.41 & 15.1 & 15.1 & $0.6 \%$ \\
$08: 55$ & 0.46 & 17.0 & 16.8 & $1.2 \%$ \\
$08: 59$ & 0.52 & 19.3 & 18.2 & $6.4 \%$ \\
$09: 02$ & 0.50 & 18.5 & 19.0 & $2.9 \%$ \\
$09: 06$ & 0.56 & 20.5 & 20.0 & $2.9 \%$ \\
\hline \multicolumn{5}{r}{} \\
& & Average: & Low flow velocity: & $13.3 \%$ \\
& & High flow velocity: & $3.0 \%$ \\
\hline
\end{tabular}

Table 3. Discharges measured in the river Sûre (10 May 2011); the absolute deviations are relative to the average discharge measured with the rising bubble technique.

\begin{tabular}{lcc}
\hline Photo & $Q\left(1 \mathrm{~s}^{-1}\right)$ & Deviation \\
\hline 1 & 665 & $0.00 \%$ \\
2 & 666 & $0.15 \%$ \\
3 & 676 & $1.65 \%$ \\
4 & 689 & $3.61 \%$ \\
5 & 638 & $4.06 \%$ \\
6 & 653 & $1.80 \%$ \\
7 & 668 & $0.45 \%$ \\
\hline Average & 665 & $1.68 \%$ \\
\hline
\end{tabular}

The discharge found with the propeller current meter is $6931 \mathrm{~s}^{-1}$, yielding a deviation of approximately $4 \%$ with respect to the rising bubble technique, which can be caused by both the limitations of the propeller current meter and the rising bubble technique. The irregular river bed can cause an inaccurate calculation of the cross-sectional area by which the propeller current meter measurements are multiplied. For the rising bubble technique, a too inaccurate measurement of the rising velocity can introduce a systematic error. An error in the rising velocity affects the calculated discharge linearly.

These first results show that the method appears valuable for discharge measurements in certain natural rivers. Further research should go more into detail on applying it under such circumstances.

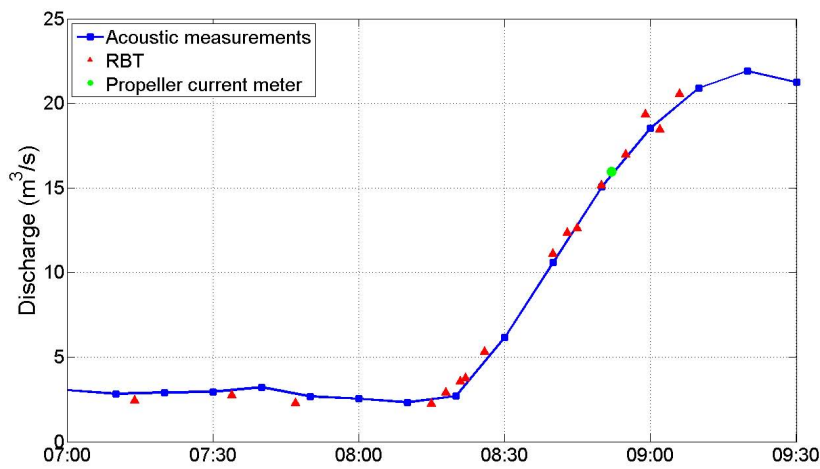

Fig. 8. Time-series of rising bubble technique (RBT) measurements plotted against acoustic discharge measurements (9 October 2009).

\subsection{Measurement errors}

Sargent (1982b) gave an elaborate description of the measurement errors involved with the rising bubble technique and Liu and Morris (1970) analyzed errors introduced by turbulence. For this reason, we will restrict ourselves to the measurement errors involved with digital image processing.

When transforming a photograph, errors are introduced by (a) information loss due to interpolating pixels, (b) inaccurate marking of the points of interest, and (c) the inaccurate marking of the reference points based on which the image is transformed. 


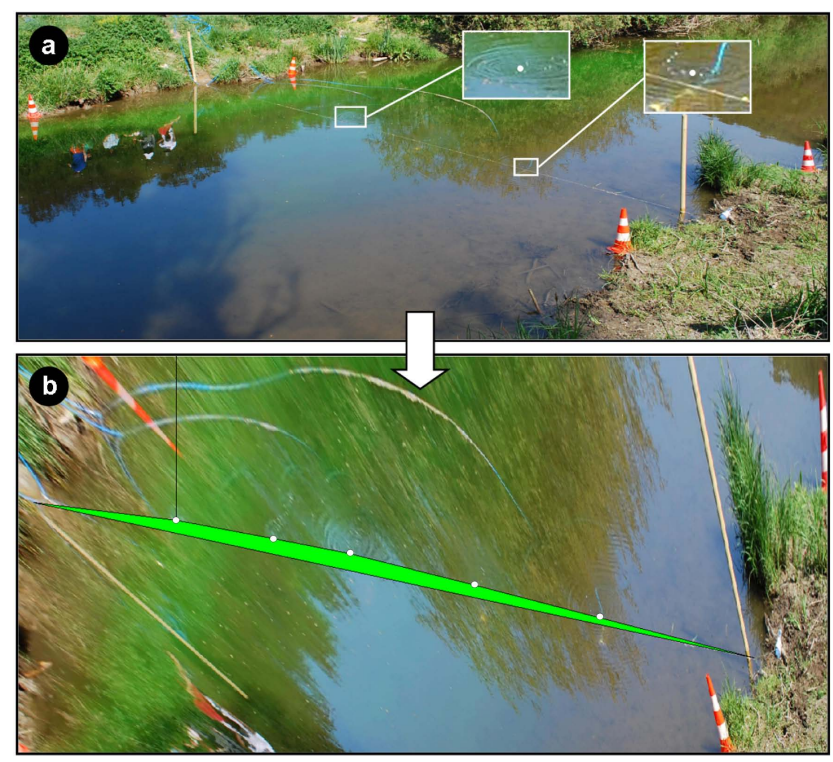

Fig. 9. Application in the river Sûre; (a) shows two examples of marked bubbles, (b) shows the final result of the image processing from which the green area $A$ can be obtained.

Error source (a) is caused by the resampling of pixels during the correction for lens distortion and the reprojection to a 2-D image, becoming significant with a large angle between the camera view and the top view to which the image is reprojected. However, we decided to already mark the points of interest before these processes, excluding the need to interpolate pixels before marking the bubbles, since these markings have double instead of integer precision.

Photographing from a high incidence angle and effects of sun and shade influence the visibility of air bubbles on the water surface and the possibility to mark them accurately. The following expands further on this error source (b).

Reflecting sunlight may complicate determining the locations where the bubbles surface, as we showed in Fig. 9a. It is recommendable to select a proper location with respect to sunlight to take photographs. If this is not possible and the sunlight heavily influences the visibility of the points of interest, a decrease in accuracy can be prevented by including a step in the image analysis as shown in Fig. 10, where the bubbles are easily observable irrespective of their locations in sun or shade. This step applies a filter to the image that calculates the standard deviation of the color value of each pixel together with that of its surrounding pixels. Large transitions are represented by a high standard deviation. This way, the resulting picture displays surfacing bubbles with light grey pixels and the transition to the shade with just a line of another color (in this case blue).

When photographing under a high incidence angle, it becomes difficult to properly mark a location, as a small shift of a mark on the picture means a relatively large shift in the location on the water surface. We expect that this error

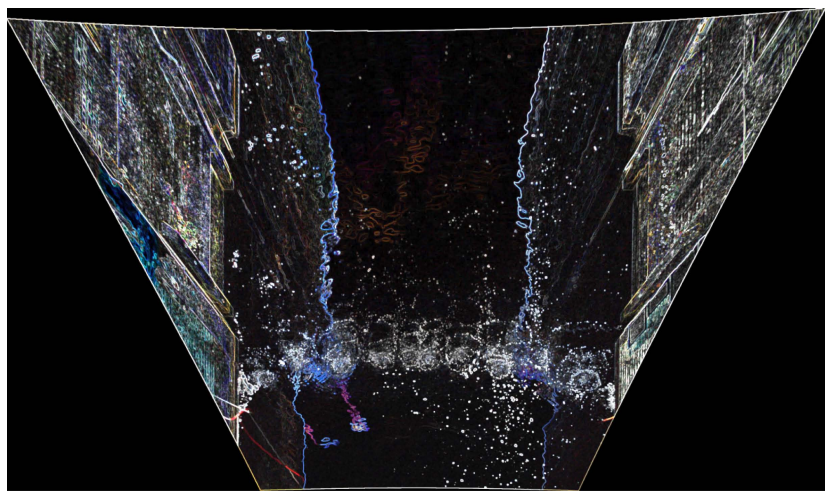

Fig. 10. Standard deviations in the color values of each pixel's neighbouring area.

source (b) is negligible in our results from the lock, since our photographs were captured from almost straight above the area between nozzle line and bubble envelope. The photographs of the river Sûre were taken from a larger angle, causing a larger source of random errors due to the possibly inaccurate marking of surfacing bubbles, nozzle line and reference points. The consistent results for the different photos suggest that even in the Sûre River case this error source was relatively small, although they are based on a limited amount of measurements. Apparently, taking photographs from moderate incidence angles is already sufficient to mitigate significant effects from this error source.

Error source (c) can play a role with an unsuitable configuration of the reference points, with an inaccurate measurement of the distances between the reference points, or with a poor distinction of the reference points in the image.

In the case studies, the reference points formed quadrilaterals that approximated a rectangle shape with a ratio of sides not larger than three times unity. The reference points were easy to distinct, although an estimation had to be made in the Surre case for two traffic cones that were a few centimeters above the water level.

In the lock, the relative distances between the reference points were measured with about $4 \mathrm{~cm}$ accuracy, yielding a relative error across the enclosed area $\left(53.6 \mathrm{~m}^{2}\right)$ that is fairly negligible. For the range of scales suiting this method, we can assume a relative error in distance measurement that remains within the same order of magnitude. For example, for our flume measurements, we could determine the relative distances with an absolute error of two millimeters which is equally negligible to the $0.72 \mathrm{~m}^{2}$ enclosed by the reference points.

\subsection{Comparison with other techniques}

The main focus of this paper is showing the value of image processing for the rising bubble technique. We show this for applications under various conditions in both a lock and a 
Table 4. Applicability of the rising bubble technique for various situations (situations in italics were actually applied).

\begin{tabular}{ll}
\hline Situation & Applicability \\
\hline Low-order streams (depth: $<25 \mathrm{~cm}$ ) & $\begin{array}{l}\text { Inaccurate method, since the low depth causes the horizontal displacement at the surface } \\
\text { to be small } \\
\text { Accurate method (when turbulence is not too high for the bubble plume to be } \\
\text { discernable); pictures can be taken from one bank with a normal resolution } \\
\text { Accurate method when pictures are taken with a higher resolution than that of a } \\
\text { commercial camera or from above the stream (from a bridge, or if the river is wadeable } \\
\text { using equipment in the river) }\end{array}$ \\
Accurate method when taking pictures at several points along the cross-section for \\
which additional reference points are needed; since nozzle installation gets more \\
complicated, application in these situations is of interest with a permanent installation \\
(in the case of a fixed set-up, reference points are only needed during its calibration) \\
Accurate method (subject to the same considerations of depth and width as in the above)
\end{tabular}

natural river. Our results can lead to an estimation of the applicability of the method for various situations, like we have listed in Table 4. Note that this table focuses on the applicability of the technique and does not focus on whether the method can be preferable in this situation (currently, we regard the method not preferable in rivers of more than $30 \mathrm{~m}$ in width and in rivers with a depth less than $25 \mathrm{~cm}$ ).

Aspects like the availability of money and time and the demanded accuracy determine whether a method is preferable. With respect to time consumption, it is quicker to use a Doppler current meter than to install nozzles and measure with the rising bubble technique. However, Doppler current meters are a lot more expensive compared to the equipment needed for the rising bubble technique. Both can be considered as relatively accurate discharge gauging techniques (of course depending on the circumstances).

If we compare the rising bubble technique with a current meter measurement, they compare in terms of initial expense. Of these, the former is more accurate by design (the air bubble integrates the horizontal velocity over the depth and all the verticals across the stream are measured instantaneously). Generally, the rising bubble technique is quicker than measurements with a current meter, certainly when the equipment and reference points are left on site, or when only the air nozzles have to be re-installed. The reason for this is that a propeller or electromagnetic sensor has to be adjusted to the right relative depths in every vertical, which is very time consuming, whereas the rising bubble technique does not even require measurements of river depth.

\section{Conclusions}

Although the integrating float method dates back to the 19th century, its full potential has never been recognized. In the past, the method was improved by Viol and Semenov (1964), who were the first to describe it using air bubbles as floats, and Sargent (1981, 1982a), who researched the practicalities of this technique. However, practical difficulties still impose a threshold for applying the technique. We show that part of these difficulties can currently be removed by applying image processing techniques.

Our results in the lock case study over time correlate well with our reference measurements. The good agreement between both methods appears particularly at higher discharges, as is also expected for this method. For lower discharges, the results deviate from the reference measurements by about $13 \%$. Further research should clarify how the technique performs under these circumstances. Experiments in a laboratory flume show that this method is very accurate under controlled circumstances. Moreover, the first results from the application of the method in a natural river look promising and show the method's value in a natural environment.

Although the rising bubble technique already appears competitive with other discharge gauging methods, we think that further improvements in the nozzles and research to their effect on the shape of air bubbles, together with other factors like water temperature, depth and contamination, may constitute a significant improvement of the technique. The main motive to search improvement in this part of the method is the linear relation of the rising velocity with the calculated discharge and the uncertainty introduced by the field measurement, which is often not an ideal situation.

We conclude that the method is applicable in relatively deep streams with an average to high flow velocity. At very high velocities, eddies caused by turbulence may affect the bubble paths too much to measure accurately. Apparently, this was not the case for the flow velocities we observed. Our results from the laboratory flume show that the analysis of a series of photographs largely mitigates the negative influence of turbulence. We believe that this is a strength of this method, since modern image processing techniques facilitate the efficient processing of multiple photographs. 
Examples of situations in which this technique can be applied are canals and moderate streams. For larger rivers, professionals are required to install the equipment. This is only profitable in cases where discharges are frequently or continuously measured.

The application in the field is simple: once the nozzles are installed, only pictures need to be taken of the water surface, each being a single measurement. This way, one can also capture the development of the discharge over time (as shown in Fig. 8).

\section{Recommendations}

In this study, we show that the rising bubble technique can compete with other discharge gauging methods in terms of costs, time consumption and accuracy. Moreover, we still see opportunities to improve the technique. This section categorizes them in two groups: improvements concerning the production of air bubbles that rise with a constant velocity and the image processing techniques.

First, more research to the proper types of air bubbles offer a large opportunity to improve the technique. The rising velocity follows from the difference between the buoyant force of the bubble and the drag force imposed on the bubble. Since the size of an air bubble increases as it rises to the surface, both forces vary as well. Interestingly, within a certain range of bubble sizes, the remainder of the forces is nearly constant, and the rising velocity hardly changes. From Janssen and Warmoeskerken (1997); Lehrer (1980); Wesselingh (1987), we find that this range is dependent on the contamination of the water, but the limits are roughly 2 and $4 \mathrm{~mm}$. When we are able to produce air bubbles that initially have sizes in the lower end of this range, the velocity will remain nearly constant as they expand while surfacing. To this end, we think that more research to the type of nozzle and the type of gas can improve the method.

Since we found that the bubbles' rising velocity during our field study differed significantly from our lab experiments, more information is needed on the influence of factors that possibly play a role here, like water temperature and contamination. More research into these influences will help to improve the accuracy of the method. When the influence appears unpredictable, we argue that a field measurement of the rising velocity for the governing circumstances will still lead to proper measurement results.

Second, the novel direction that we choose with respect to image processing offers opportunities for further improvement. The statistical procedure applied in Fig. 10 can also help automate the complete process of obtaining a discharge from a photograph. As the figure shows, patterns are easily recognizable and current pattern recognition techniques allow us to find these patterns automatically. Using pattern recognition techniques to completely automate the process requires further research.
Following from these recommendations, the rising bubble technique has the potential to become a good alternative for discharge measurement techniques in moderate rivers and canals.

\section{Supplementary material related to this article is available online at: http://www.hydrol-earth-syst-sci.net/16/345/2012/ hess-16-345-2012-supplement.zip.}

Acknowledgements. The authors wish to thank H. H. G. Savenije, T. A. Bogaard, A. M. J. Coenders-Gerrits, R. J. van der Ent and S. A. P. de Jong for their ideas and dedicated support and the Water board Noorderzijlvest for providing reference discharge data.

Edited by: M. Weiler

\section{References}

Aybers, N. and Tapucu, A.: The motion of gas bubbles rising through stagnant liquid, Wärme. Stoffübertrag., 2, 118-128, 1969a.

Aybers, N. and Tapucu, A.: Studies on the drag and shape of gas bubbles rising through a stagnant liquid, Wärme. Stoffübertrag., 2, 171-177, 1969b.

Brown, D.: Decentering Distortion of Lenses, Photogramm. Eng., 32, 444-462, 1966.

Brown, D.: Close- range camera calibration, Photogramm. Eng., 37, 855-866, 1971.

De Villiers, J., Leuschner, F., and Geldenhuys, R.: Centi-pixel accurate real-time inverse distortion correction, in: Proceedings of SPIE, 7266, 726611-1-726611-8, 2008.

Devernay, F. and Faugeras, O.: Automatic calibration and removal of distortion from scenes of structured environments, in: Proceedings of SPIE, 2567, 62-72, 1995.

Devernay, F. and Faugeras, O.: Straight lines have to be straight, Mach. Vision Appl., 13, 14-24, 2001.

Fryer, J. and Brown, D.: Lens distortion for close-range photogrammetry, Photogramm. Eng. Rem. S., 52, 51-58, 1986.

Hajos, S.: Neues Verfahren zur Messung kleiner Wassergeschwindigkeiten, Zentralbl. Bauverwaltung, 24, 281-283, 1904 (in German).

Herschy, R.: Hydrometry; principles and practices, Wiley Interscience, 1978.

Herschy, R.: Streamflow measurement, Taylor \& Francis, 1995.

Janssen, L. and Warmoeskerken, M.: Transport phenomena data companion, Delftse Universitaire Pers., 1997.

Lehrer, J.: A theoretical criterion of transition in the free motion of single bubbles and drops, AIChE J., 26, 170-172, 1980.

Liu, H. and Morris, C. D.: Integrating-float measurements in turbulent flows, J. Hydr. Eng. Div.-ASCE, 96, 565-572, 1970.

Neale, W., Hessel, D., and Terpstra, T.: Photogrammetric Measurement Error Associated with Lens Distortion, in: Accident Reconstruction, 2011, SAE International, 2011-01-0286, doi:10.4271/2011-01-0286, 2011.

Nowakowski, A. and Skarbek, W.: Lens radial distortion calibration using homography of central points, in: EUROCON, 2007, 
the International Conference on "Computer as a Tool”, 340-343, IEEE, 2007.

Sargent, D.: Development of a viable method of stream flow measurement using the integrating float technique, P. I. Civil Eng. Pt. 2, 71, 1-15, 1981.

Sargent, D.: Rising air float technique for the measurement of stream discharge, in: Advances in hydrometry, no. 134 in IAHS Publ., 153-164, IAHS-AISH P., Exeter, Engl., 1982a.

Sargent, D.: The accuracy of streamflow measurements using the rising air float technique., P. I. Civil Eng. Pt. 2, 73, 179-182, 1982b.

Sargent, D. and Davis, T.: Discussion: The development of a viable method of stream flow measurement using the integrating float technique, P. I. Civil Eng. Pt. 2, 71, 949-950, 1981.

Shaw, E.: Hydrology in practice, Routledge, 1994.

Stein, G.: Lens distortion calibration using point correspondences, in: Proceedings of the IEEE Computer Society Conference on Computer Vision and Pattern Recognition, 602-608, IEEE, Los Alamitos, CA, United States, San Juan, PR, USA, 1997.

Swaminathan, R. and Nayar, S.: Nonmetric calibration of wideangle lenses and polycameras, Pattern Analysis and Machine Intelligence, IEEE T. Pattern. Anal., 22, 1172-1178, 2000.
Toop, C., Webster, P., and Hawnt, R.: Improved guidelines for the use of the rising air float technique for river gauging, J. Chart. Inst. Water E., 11, 61-66, 1997.

Tsai, R. Y.: Versatile Camera Calibration Technique for HighAccuracy 3D Machine Vision Metrology Using Off-the-Shelf TV Cameras and Lenses, IEEE Robot. Autom. Mag., RA-3, 323344, 1987.

Viol, V. and Semenov, V.: Experiments in measuring discharges in canals by the photo-integration method, Soviet Hydrol. Selected Pap., 2, 198-199, 1964.

Wesselingh, J.: The velocity of particles, drops and bubbles, Chem. Eng. Process.: Process Intensification, 21, 9-14, 1987.

Wolberg, G., of Electrical, I., and Society, E. E. C.: Digital image warping, vol. 3, IEEE Computer Society Press New York, 1990.

Yannopoulos, P., Demetracopoulos, A., and Hadjitheodorou, C.: Quick method for open-channel discharge measurements using air bubbles, J. Hydraul. Eng.-ASCE, 134, 843-846, 2008. 